\section{Maltrato infantil: ¿conocer su impacto económico favorece su prevención?}

\section{Child abuse: does knowing the economic impact favors its prevention?}

Una patología que ha existido desde tiempo inmemorial en la humanidad es la violencia que sufren las niñas, niños y adolescentes. En 1989, en la Asamblea General de la Organización de las Naciones Unidas, se dio una atención muy especial a este problema y se enfatizó el interés superior por este grupo etario con el objetivo de evitar que se afecten sus integridades física, emocional, educativa y económica, entre otras.

Dentro de esta terrible problemática destaca una patología médica, el maltrato infantil, reconocida ampliamente como tal desde mediados del siglo XX, cuyo interés y conocimiento se ha incrementado notablemente en muchos países del mundo, incluyendo a México. En 1999 la Organización Mundial de la Salud la clasificó como un problema de salud pública mundial.

En nuestro medio se ha insistido en la importancia de considerar esta situación como un problema médico-social-legal. La idea es concientizar a todo profesional o adulto que atienda o interactúe con personas de este grupo etario para que pueda sospechar, diagnosticar y atender integralmente a la víctima y a su familia cuando, desgraciadamente, haya ocurrido el pro- blema. Sin embargo, en México los avances son lentos y en general no cubren las expectativas que existen en otros países a pesar de algunos esfuerzos aislados, incluyendo los emanados de la Presidencia de la República para cumplir con los compromisos adquiridos con la comunidad internacional desde hace aproximadamente 25 años.

Un enfoque que quizá podría despertar las conciencias de los secretarios de Salud, de Educación, de Desarrollo Social, gobernantes, empresarios y administradores, sería establecer un cambio de paradigma en la atención de este problema. Ello puede ocurrir si se señala el impacto económico y social que el maltrato ocasiona en el menor agredido, en su familia, en la sociedad en general y, como consecuencia, el desarrollo de un verdadero enfoque prioritario al plantear estrategias factibles de prevención primaria.

Para puntualizar el primer enfoque es menester mencionar algunos aspectos básicos del problema tales como su frecuencia, así como las consecuencias físicas y emocionales en la víctima, como se ha hecho en algunos países con economías líderes. 
En Estados Unidos se ha visto que en la última década se han detectado, anualmente, aproximadamente un millón de casos de alguna modalidad de maltrato infantil; de ellos, entre 100,000 y 200,000 son víctimas de una agresión craneal intencional y han fallecido entre 193 y 367 de cada 100,000 menores.

En México, aunque no existen cifras precisas, se puede decir que este problema es evidente con base en el número creciente de casos extremos atendidos en diversas instituciones gubernamentales, pero básicamente en centros hospitalarios pediátricos de tercer nivel en las dos últimas décadas.

El segundo punto es señalar el costo económico que implica la atención a cada una de las niñas, niños o adolescentes víctimas. Para ello es necesario determinar los costos directos (la suma del personal profesional involucrado y los gastos de hospital en la atención integral de cada caso) e indirectos (disminución en el aprendizaje, baja productividad, limitación en el trabajo que puede tener la víctima, así como el tiempo invertido por los padres o cuidadores del menor y la pérdida sus de salarios).

Una estimación hecha en el 2010, en Estados Unidos, mostró que el costo promedio por tiempo de vida de una víctima de maltrato infantil no fatal fue de 210,012 dólares. El desglose de este costo incluye 32,648 en atención de la salud en la edad infantil; 10,530 en atención médica de la edad adulta; 144,360 en la pérdida de su productividad; 7,728 en servicios de bienestar infantil; 6,747 en justicia criminal y 7,999 en costos de educación especial. El costo estimado por vida perdida fue calculado en 1,272,900 dólares. La carga económica a lo largo de la vida, entre los nuevos casos fatales y no fatales, es de aproximadamente 124 mil millones de dólares. En total la carga económica se estimó en 585 mil millones de dólares que, comparada con la de otros problemas de salud, evidencia un impacto económico sustancial.

En Alemania el gasto por atender las diversas modalidades del maltrato infantil asciende a 11.1 billones de euros $y$, para las consecuencias a mediano y corto plazos, ascienden a 29.8 billones.

En México, un informe periodístico confiable señaló que la atención inmediata y las consecuencias tardías de los daños generados por el maltrato infantil son aproximadamente de $20 \mathrm{mil}$ millones de pesos anuales para las Instituciones del Gobierno Federal. En el mismo informe se estima que para el año 2050 el gasto ascenderá a 147,839 millones de pesos si no se destinan recursos para mejorar la identificación, atención y prevención de esta patología.

En un estudio exploratorio y parcialmente realizado en la Coordinación de Estudios Avanzados sobre Maltrato Infantil-Prevención del Instituto Nacional de Pediatría (CEAMI-P-INP), con base en 14 niños que fueron diagnosticados $y$ atendidos por haber sufrido síndrome del niño sacudido, forma extrema de abuso físico infantil, se analizaron los datos correspondientes a los diversos servicios médicos y paramédicos empleados por el instituto. Durante la hospitalización de cada paciente se incluyeron los medicamentos requeridos, las curaciones, la intervención de los médicos residentes y del personal de enfermería, excepto la alimentación de los menores. En ese análisis se consideraron también los exámenes de laboratorio y gabinete realizados.

Para precisar el costo económico se empleó el tabulador de cuotas del Instituto Nacional de Pediatría en el nivel socioeconómico 6, que conlleva $100 \%$ de cada procedimiento; éste se toma como base para reducir los niveles inferiores de acuerdo con los porcentajes de subsidio que el Gobierno Federal aporta en cada nivel. Los resultados obtenidos, que aún no se han publi- 
cado, revelaron un costo aproximado, de todos los casos, de 942,965.00 pesos; o sea 67,355 pesos por paciente. Estos resultados, aunque no fueron comparados con el gasto causado en otros pacientes con patologías diferentes y en el mismo período de hospitalización, sí resultan francamente elevados. Obviamente, en este estudio no se consideraron los gastos implícitos en la recuperación o en la rehabilitación física y emocional; el impacto emocional y económico en la familia y la consecuencia económica y social en la comunidad. Sin embargo, y a pesar de las diferencias establecidas en este estudio con otras publicaciones internacionales, evidentemente el costo económico sigue siendo muy elevado.

Por ello, se remarca la importancia de prevenir o por lo menos disminuir la elevada prevalencia del maltrato infantil en nuestro país mediante la implementación de estrategias verdaderamente factibles y de aplicación nacional. En este sentido, en la CEAMI-P-INP se han propuesto diversas líneas de acción y de investigación con base en la difusión y aplicación de los Derechos de las Niñas, Niños y Adolescentes; el desarrollo e implementación de una crianza humanizada sostenida por un programa de puericultura eficaz en todo el país. Apoyar las diferentes campañas existentes que pregonan y luchan por una cultura de paz nacional; el impulso de proyectos de investigación para crear e implementar herramientas que permitan detectar menores víctimas de diferentes modalidades de maltrato, pero que por la severidad del daño no requieren atención médica ya que no lo señalan por miedo o porque creen que así es la vida. Así mismo, desarrollar programas de investigación con al- cance nacional que permitan detectar adultos "potencialmente" maltratadores y que funcionan en la sociedad como personas aparentemente normales; considerar que un buen número de ellos tienen la responsabilidad de atender niñas, niños y adolescentes.

Debe quedar muy claro que en este programa la investigación y las funciones de las autoridades gubernamentales y de la sociedad civil deben estar unidas, para establecer un paradigma de atención integral y de prevención en el que la negligencia, la corrupción y la impunidad no tengan lugar.

Finalmente, ¿será posible que si se conoce el impacto económico que genera la atención médica de estos pacientes, permitirá despertar el interés por la prevención de este problema? Seguramente la implementación de estrategias con este enfoque debería ser la estrategia a seguir, puesto que de esta manera se tiene un menor costo humano y económico.

La tarea es ardua pero la niñez mexicana merece el esfuerzo y el compromiso de todas las personas que tienen la responsabilidad de que ellos alcancen a plenitud su autonomía, autoestima, creatividad, felicidad, educación, solidaridad y salud. De otra manera, este grupo etario seguirá siendo víctima de todas las adversidades sociales en boga y víctima de la violencia existente en sus diversas modalidades.

Dr. Arturo Loredo-Abdalá Investigador D. Institutos Nacionales de Salud Coordinador de la Coordinación de Estudios Avanzados sobre Maltrato Infantil- Prevención 